Pacific Journal of Mathematics

AN ASYMPTOTIC PROPERTY OF THE EULER FUNCTION 


\title{
AN ASYMPTOTIC PROPERTY OF THE EULER FUNCTION
}

\author{
R. J. MIECH
}

Let $\varphi(n)$ denote the Euler function. The starting point of this paper is the simple observation that if $p$ is a prime then $p$ and $\varphi(p)+1=p$ have a common divisor which is greater than 1; its conclusion is: if $\left\{m_{k}\right\}$ is the sequence of positive square free integers which have $k$ prime factors, where $k \geqq 2$, then the number of integers $m_{k}$ not exceeding $x$ such that $m_{k}$ and $\varphi\left(m_{k}\right)+1$ have a common divisor other than 1 is asymptotic to

$$
\lambda_{k} \frac{x}{\log x}(\log \log x)^{k-2},
$$

where $\lambda_{k}$ is a positive constant that depends on $k$.

The source of the problem under consideration was a question raised by Gordon in the course of his investigations of Hajós factorization of abelian groups. The question was: are there integers $n$, other than primes and their doubles, such that $\varphi(n)+1$ divides $n$. This is still an open problem. However, if we relax our demands, as we have done above, it is possible to prove the asymptotic relation stated there.

One of the main results needed to establish the first assertion of this paper is:

Lemma 1. Let $a$ be a positive integer and $b_{1}, \cdots, b_{k}$ be a set of integers such that $0<b_{i}<a$ and $\left(b_{i}, a\right)=1$ for $i=1,2, \cdots, k$. Let $b_{1}^{\prime}, \cdots, b_{q}^{\prime}$ denote the distinct integers which appear in the sequence $b_{1}, \cdots, b_{k}$ and suppose that $b_{j}^{\prime}$ appears $r_{j}$ times for $j=1,2, \cdots, q$. Let $\pi\left(x ; a, b_{1}, \cdots, b_{k}\right)$ denote the number of square free integers $n$ not exceeding $x$ such that $n=p_{1} p_{2} \cdots p_{k}$ where $p_{i}$ is a prime and $p_{i} \equiv b_{i} \bmod a$ for $i=1,2, \cdots, k$. Then for $k \geqq 2$ we have

$$
\pi\left(x ; a, b_{1}, \cdots, b_{k}\right)=\frac{1}{r_{1} ! \cdots r_{q} !} \frac{k}{\phi^{k}(a)} \frac{\log _{2}^{k-1} x}{\log x}\left[1+\mathcal{O}\left(\frac{\log _{3} x}{\log _{2} x}\right)\right]
$$

uniformly for $a \leqq \log _{k+1} x$, where $\log _{j} x$ is the jth iterated logarithm of $x$. The constant implied by the O-term depends on $k$.

The proof of Lemma 1 is based on a generalization of one of Wright's ideas [2]. We begin this proof by listing several known results about primes in arithmetic progressions. 
Lemma 2. Let $\pi(x ; a, b)$ denote the number of primes not exceeding $x$ which are congruent to $b$ modulo $a$, where $0<b<a$ and $(a, b)=1$. Then if

$$
\alpha \leqq \exp \left[c_{1} \log x / \log _{2} x\right],
$$

where $c_{1}$ is an absolute constant, we have

$$
\pi(x ; a, b)=\frac{1}{\varphi(a)} \frac{x}{\log x}\left[1+\varnothing\left(\frac{1}{\log x}\right)\right]
$$

except, possibly, for a set of integers $\left\{a^{\prime}\right\}$ all of which are multiples of a single integer $\alpha^{\prime \prime}$ which, in turn, is greater than $\log ^{4} x$, where $A$ is any fixed positive constant. The constant implied by the O-term depends on $A$.

See Chapter 9, Theorem 2.3 of [1] for a proof.

Frequent use will be made of the following form of this lemma: if $\log x \leqq u$ and $a \leqq \log _{2} x$ then

$$
\pi(u ; a, b)=\frac{1}{\varphi(\alpha)} \frac{u}{\log u}\left[1+\mathcal{O}\left(\frac{1}{\log u}\right)\right],
$$

where the constant implied by the $O$-term is independent of $u$ and $x$. We shall also employ:

LEMma 3. If $a<x$ then there is an absolute constant $c_{2}$ such that

$$
\pi(x ; a, b) \leqq \frac{c_{2}}{\varphi(a)} \frac{x}{\log (x / a)}
$$

See [1], Chapter 2, Theorem 4.1 for a proof.

The next lemma is a straightforward consequence of Lemmas 2 and 3 .

Lemia 4 . If $a \geqq 27,0<b<a$ and $(a, b)=1$ then

$$
\sum_{\substack{p \leq x \\ p=b \bmod a}} \frac{1}{p}=\frac{1}{\varphi(a)} \log _{2} x+\mathcal{C}\left(\frac{\log _{3} x}{\varphi(a)}\right)
$$

uniformly for all integers $a \leqq 2 \log _{3} x$.

The balance of this section deals with the proof of Lemma 1. As for notation, $a, b_{1}, \cdots$, and $b_{k}$ will be the integers defined in Lemma 1 , any prime $p_{i}$ which occurs will be congruent to $b_{i}$ modulo $a$ for $i=1,2, \cdots, k$, and a prime on a summation symbol (as in $\Sigma^{\prime}$ ) will 
indicate that any prime $p_{i}$ appearing in the index of summation is congruent to $b_{i}$ modulo $a$. The symbols $c_{1}, c_{2}, \cdots$ will denote constants that depend, at most, on $k$. We also assume that $k \geqq 2$.

LEMMA 5. Let

$$
L\left(x ; a, b_{1}, \cdots, b_{k}\right)=\sum_{p_{1} \cdots p_{k} \leqq x}^{\prime} \frac{1}{p_{1} \cdots p_{k}}
$$

where the primes $p_{i}$ run independently through the residue classes $b_{i}$. Then if $x \geqq c_{3}$ we have

$$
L\left(x ; a, b_{1}, \cdots, b_{k}\right)=\frac{1}{\phi^{k}(a)} \log _{2}^{k} x\left[1+\mathcal{O}\left(\frac{\log _{3} x}{\log _{2} x}\right)\right]
$$

uniformly for $a \leqq \log _{3} x$.

Proof. Since

$$
\prod_{i=1}^{k}\left(\sum_{p_{i} \leqq x^{1 / k}}^{\prime} \frac{1}{p_{i}}\right) \leqq L\left(x ; a, b_{1}, \cdots, b_{k}\right) \leqq \prod_{i=1}^{k}\left(\sum_{p_{i} \leqq x}^{\prime} \frac{1}{p_{i}}\right)
$$

and since $a$ is chosen so that

$$
a \leqq \log _{3} x \leqq 2 \log _{3} x^{1 / k},
$$

Lemma 5 follows from Lemma 4.

LEMMA 6. Let

$$
\vartheta\left(x ; a, b_{1}, \cdots b_{k}\right)=\sum_{p_{1} p w_{k} \leqq x}^{\prime} \log p_{1} \cdots p_{k}
$$

where the primes $p_{i}$ run independently through the residue classes $b_{i .}$ Then if $x>c_{4}$ and $a \leqq \log _{k+1} x$ we have

$$
\vartheta\left(x ; a, b_{1}, \cdots, b_{k}\right)=\frac{k}{\phi^{k}(a)} x \log _{2}^{k-1} x\left[1+\mathcal{O}\left(\frac{\log _{3} x}{\log _{2} x}\right)\right] .
$$

The proof is an inductive one. For $k=2$ we have

$$
\vartheta\left(x ; a, b_{1}, b_{2}\right)=\sum_{p_{2} \leqq x}^{\prime} \sum_{p_{1} \leqq x / p_{2}}^{\prime} \log p_{1}+\sum_{p_{2} \leqq x}^{\prime} \sum_{p_{1} \leqq x / p_{1}}^{\prime} \log p_{2} .
$$

Now, the first double sum on the right hand side of this equation can be expressed as the sum of three double sums, $\Sigma_{1}, \Sigma_{2}, \Sigma_{3}$ whose indices of summation are

$$
\begin{gathered}
p_{2} \leqq x / \log x p_{1} \leqq \log x, \quad p_{2} \leqq x / \log x \quad \log x<p_{1} \leqq x / p_{2} \\
x / \log x<p_{2} \leqq x \quad p_{1} \leqq x / p_{2},
\end{gathered}
$$

respectively. Let us consider $\sum_{2}$ first. We have, by Lemma 2 


$$
\begin{aligned}
& \sum_{\log x<p_{1} \leqq x / p_{2}}^{\prime} \log p_{1} \\
& \quad=\frac{1}{\varphi(\alpha)}\left[\frac{x}{p_{2}}-\log x+\infty\left(\frac{x}{p_{2}} \frac{1}{\log _{2} x}\right)\right] .
\end{aligned}
$$

Morecver, since $2 \log _{3}(x / \log x) \geqq \log _{3} x \geqq a$, Lemma 4 can be applied. Doing so we find that

$$
\sum_{p_{2} \leqq x / \log x}^{\prime} \sum_{\log }^{\prime} \sum_{x<p_{1} \leqq x / p_{2}}^{\prime} \log p_{1}=\frac{x}{\varphi^{2}(\alpha)} \log _{2} x\left[1+\mathcal{O}\left(\frac{\log _{3} x}{\log _{2} x}\right)\right] .
$$

A straightforward application of Lemma 3 will lead us to tolerable bounds for $\Sigma_{1}$ and $\Sigma_{3}$. If we do this and then apply the same argument to the second double sum that appears in the equation for $\vartheta\left(x ; a, b_{1}, b_{2}\right)$ we will have our result for $k=2$.

Let us go on to the induction. Set

$$
\begin{aligned}
& f\left(x / p_{i}\right)=\vartheta\left(x / p_{i} ; a, b_{1}, \cdots, b_{i-1}, b_{i+1}, \cdots, b_{k+1}\right) \quad \text { for } \quad 1 \leqq i \leqq k+1, \\
& g\left(x / p_{i}\right)=L\left(x / p_{i} ; a, b_{1}, \cdots, b_{i-1}, b_{i+1}, \cdots, b_{k}\right) \quad \text { for } \quad 1 \leqq i \leqq k \\
& g\left(x / p_{k+1}\right)=L\left(x / p_{k+1} ; a, b_{2}, \cdots, b_{k}\right)
\end{aligned}
$$

and

$$
h\left(x / p_{i}\right)=f\left(x / p_{i}\right)-(k / \varphi(a))\left(x / p_{i}\right) g\left(x / p_{i}\right) \quad \text { for } \quad 1 \leqq i \leqq k+1 .
$$

Then, since

and since for $1 \leqq i \leqq k$,

$$
k \vartheta\left(x ; a, b_{1}, \cdots, b_{k+1}\right)=\sum_{i=1}^{k+1} \sum_{p_{i} \leqq x}^{\prime} f\left(x / p_{i}\right)
$$

$$
L\left(x ; a, b_{1}, \cdots, b_{k}\right)=\sum_{p_{i} \leqq x}^{\prime}\left(1 / p_{i}\right) g\left(x / p_{i}\right),
$$

it follows that

$$
\begin{aligned}
k \vartheta\left(x ; a, b_{1}, \cdots, b_{k+1}\right) & -\frac{k^{2}}{\varphi(a)} x L\left(x ; a, b_{1}, \cdots, b_{k}\right) \\
& -\frac{k}{\varphi(a)} x L\left(a, b_{2}, \cdots, b_{k+1}\right) \\
= & \sum_{i=1}^{k+1} \sum_{p_{i} \leqq x}^{\prime} h\left(x / p_{i}\right) .
\end{aligned}
$$

Now, if $p_{i} \leqq x / \log x$ then $x / p_{i} \geqq \log x$ and $\log _{k+1}\left(x / p_{i}\right) \geqq \log _{k+2} x \geqq a$. Thus, by the inductive hypothesis and Lemma 5, we have

$$
\sum_{p_{i} \leqq x / 103 x}^{\prime} h\left(x / p_{i}\right)=\mathcal{O}\left[\frac{x}{\phi^{k+1}(a)}\left(\log _{3} x\right) \log _{2}^{k-1} x\right] \text {. }
$$

If, on the other hand, we have $x / \log x<p_{i} \leqq x$ then $x / p_{i} \leqq \log x$, and we can show, by referring to the definitions of the quantities 
involved, that

$$
\sum_{x / \log } \sum_{x<p_{i} \leqq x}^{\prime} f\left(x / p_{i}\right)=\mathcal{O}\left[\frac{x}{\phi^{k+1}(a)}\left(\log _{3} x\right) \log _{2}^{k-1} x\right]
$$

and

$$
\sum_{x / \log }^{\prime} \frac{k}{x<p_{i} \leqq x} \frac{x}{\varphi(a)} \frac{x}{p_{i}} g\left(x / p_{i}\right)=\mathscr{O}\left[\frac{x}{\phi^{k+1}(\alpha)}\left(\log _{3} x\right) \log _{2}^{k-1} x\right] .
$$

In short, we have

$$
\begin{aligned}
k \vartheta\left(x ; a, b_{1}, \cdots, b_{k+1}\right) & =\frac{k^{2}}{\varphi(a)} x L\left(x ; a, b_{1}, \cdots, b_{k}\right) \\
& +\frac{k x}{\varphi(a)} L\left(x ; a, b_{2}, \cdots, b_{k+1}\right) \\
& +\varnothing\left[\frac{k}{\varphi^{k+1}(a)} \log _{3} x \log _{2}^{k-1} x\right] .
\end{aligned}
$$

Lemma 6 follows from this formula and Lemma 5.

LEMma 7. Let $d\left(n ; a, b_{1}, \cdots, b_{k}\right)$ be the number of representations of the integer $n$ of the form $n=p_{1} \cdots p_{k}$ where $p_{i} \equiv b_{i} \bmod a$ for $i=1, \cdots, k$ and the primes $p_{i}$ run independently through the residue classes $b_{i}$. Then we have

$$
\sum_{n \leqq x} d\left(n ; a, b_{1}, \cdots, b_{k}\right)=\frac{k}{\varphi^{k}(a)} x \frac{\log _{2}^{k-1} x}{\log x}\left[1+\mathcal{O}\left(\frac{\log _{3} x}{\log _{2} x}\right)\right]
$$

uniformly for $a \leqq \log _{k+1} x$.

Proof. Set $d_{k}(n)=d\left(n ; a, b_{1}, \cdots, b_{k}\right)$. Then we have

$$
\begin{aligned}
\vartheta\left(x ; a, b_{1}, \cdots, b_{k}\right)= & \sum_{n \leqq x} d_{k}(n) \log n=\left(\sum_{n \leqq x} d_{k}(n)\right) \log x \\
& -\int_{2}^{x}\left(\sum_{n \leqq u} d_{k}(n)\right) d(\log u) .
\end{aligned}
$$

Since $d_{k}(n) \leqq k !$ and since $d_{k}(n)$ is positive only if $n \equiv b_{1} \cdots b_{k} \bmod$ $a$ it follows that

$$
\sum_{n \leqq u} d_{k}(n) \leqq k !\left(\frac{u}{\varphi(a)}+1\right)
$$

Thus

$$
\int_{2}^{x}\left(\sum_{n \leqq u} d_{k}(n)\right) d(\log u) \leqq \frac{c_{5} x}{\varphi(a)} \leqq \frac{c_{6} x}{\varphi^{k}(a)}\left(\log _{3} x\right)\left(\log _{2}^{k-2} x\right) .
$$

These results, along with Lemma 6, give us Lemma 7 .

We are now in a position to prove Lemma 1 . Set

$$
e\left(n ; a, b_{1}, \cdots, b_{k}\right)=\mu^{2}(n) d\left(n ; a, b_{1}, \cdots, b_{k}\right),
$$


where $\mu(n)$ is the Möbius function. Then

$$
\begin{aligned}
0 & \leqq \sum_{n \leqq x} d\left(n ; a, b_{1}, \cdots, b_{k}\right)-\sum_{n \leqq x} e\left(n ; a, b_{1}, \cdots, b_{k}\right) \\
& \leqq \sum_{i=1}^{k} \sum_{n \leqq x} d\left(n ; a, b_{1}, \cdots, b_{i-1}, b_{i+1}, \cdots, b_{k}\right) \leqq \frac{c_{6} x}{\varphi^{k-1}(a)} \frac{\log _{2}^{k-2} x}{\log x} .
\end{aligned}
$$

Consequently

$$
\sum_{n \leqq x} e\left(n ; a, b_{1}, \cdots, b_{k}\right)=\frac{k x}{\varphi^{k}(a)} \frac{\log _{2}^{k-1} x}{\log x}\left[1+\mathcal{O}\left(\frac{\log _{3} x}{\log _{2} x}\right)\right] .
$$

This completes the proof of Lemma 1 since

$$
\pi\left(x ; a, b_{1}, \cdots, b_{k}\right)=\frac{1}{r_{1} ! \cdots r_{q} !} \sum_{n \leqq x} e\left(n ; a, b_{1}, \cdots, b_{k}\right) .
$$

2. In this section we shall prove the assertion made in the introduction of this paper. To that end, let $\Phi^{\prime}(k, x)$ denote the number of integers in the set

$$
\left\{m: 1<m \leqq x, m=p_{1}, \cdots, p_{k}, \mu^{2}(m)=1,(m, \varphi(m)+1)>1\right\},
$$

and let $\Phi(k, x)$ be the number of odd integers counted by $\Phi^{\prime}(k, x)$. Let $n_{j}$ be a generic symbol for an odd positive square free integer which has $j$ prime factors, for $j=1, \cdots, k$.

Our first goal is formula (3) below. Suppose we have $n=n_{k}=$ $p_{1} \cdots p_{k}, d \mid(n, \varphi(n)+1)$, and $d>1$; then $d$ is a square free integer which has $j$ prime factors where $1 \leqq j \leqq k-1$. Thus if we set

$$
A\left(n_{j}\right)=\left\{n_{k-j}: n_{j} n_{k-j} \leqq x,\left(n_{j}, n_{k-j}\right)=1, \varphi\left(n_{j} n_{k-j}\right)+1 \equiv 0 \bmod n_{j}\right\}
$$

an elementary combinatorial argument will yield the equation

$$
\Phi(k, x)=\sum_{j=1}^{k-1}(-1)^{j+1} \sum_{n_{j} \leqq x} \sum_{n_{k-j} \in A\left(n_{j}\right)} 1 .
$$

Consider next the quantity

$$
\sum_{\log _{k+1}} \sum_{x<n_{j} \leqq x} 1 \text {. }
$$

Since we have $n_{j}=p_{1} \cdots p_{j}$ at least one of the prime factors of $n_{j}$, say $p_{1}$, must be greater than $\left(\log _{k+1} x\right)^{1 / j}=z(j, x)$. Moreover, if $n_{j}$ and $n_{k-j}$ are relatively prime integers such that

$$
\varphi\left(n_{j} n_{k-j}\right)+1 \equiv 0 \bmod n_{j}
$$

we then have $n_{j} n_{k-j}=p_{1} n_{k-1}$ and

$$
\varphi\left(p_{1} n_{k-1}\right)+1 \equiv 0 \bmod p_{1} .
$$


Consequently the quantity (1) is bounded above by

$$
\left(\begin{array}{l}
k \\
j
\end{array}\right) \sum_{p_{1}>z(j, x)} \sum_{n_{k-1} \in A\left(p_{1}\right)} 1,
$$

for any integer $n_{j} n_{k-j}$ which appears in (1) will appear at most $\left(\begin{array}{l}k \\ j\end{array}\right)$ times in (1) and at least once in the double sum of (2). If we set $z=z(k, x)=\left(\log _{k+1} x\right)^{1 / k}$ we have

$$
\Phi(k, x)=\sum_{j=1}^{k-1}(-1)^{j+1} \sum_{n_{j} \leqq \log _{k+1}} \sum_{n_{k-j} \in A\left(n_{j}\right)} 1+\mathcal{O}(S),
$$

where

$$
S=\sum_{p_{1}>z} \sum_{n_{k-1} \in A\left(n_{1}\right)} 1
$$

We shall now show that if $k \geqq 2$ then

$$
\begin{aligned}
& \sum_{j=1}^{k-1}(-1)^{j+1} \sum_{n_{j} \leqq \log _{k+1}} \sum_{n_{k-j} \in A\left(n_{j}\right)} 1 \\
& \quad=\alpha_{k} x \frac{\log _{2}^{k-2}}{\log x}\left[1+\mathcal{O}\left(\frac{1}{\log _{k+1} x}\right)\right],
\end{aligned}
$$

where $\alpha_{k}$ is a constant that depends on $k$.

Consider any fixed $n_{j}$ which appears in (4). If $n_{k-j} \in A\left(n_{j}\right)$ then $\left(n_{k-j}, n_{j}\right)=1$ and $\varphi\left(n_{j}\right) \varphi\left(n_{k-j}\right)+1 \equiv 0 \bmod n_{j}$. Thus, if $\left(n_{j}, \varphi\left(n_{j}\right)\right)>1$ the set $A\left(n_{j}\right)$ is empty. On the other hand if $\left(\varphi\left(n_{j}\right), n_{j}\right)=1$, and $n_{k-j}=p_{j+1} \cdots p_{k}$, we have the congruence

$$
\left(p_{j+1}-1\right) \cdots\left(p_{k}-1\right) \equiv l\left(n_{j}\right) \bmod n_{j}
$$

where $l\left(n_{j}\right)$ is chosen so that $l\left(n_{j}\right) \varphi\left(n_{j}\right) \equiv-1 \bmod n_{j}$. Furthermore, if $p_{j+1} \cdots p_{k}$ is a set of primes that satisfies (5) then there is a set of integers $l_{j+1}, \cdots, l_{k}$ such that

$$
\begin{gathered}
l_{j+1} \cdots l_{k} \equiv l\left(n_{j}\right) \bmod n_{j} \\
\left(1+l_{i}, n_{j}\right)=1 \text { for } i=j+1, \cdots, k,
\end{gathered}
$$

for we need only take $l_{i}$ so that $p_{i} \equiv 1+l_{i} \bmod n_{j}$. Conversely, if $l_{j+1}, \cdots$, and $l_{k}$ are integers which satisfy $(6)$ and $(7)$ then there are primes $p_{j+1}, \cdots, p_{k}$ which satisfy (5). Note also that the number of distinct solutions of (6), where two solutions, $l_{j+1}, \cdots, l_{k}$ and $l_{j+1}^{\prime}, \cdots, l_{k}^{\prime}$, are said to be the same if and only if both contain the same integers modulo $a$ to the same multiplicity, obviously does not exceed $\varphi^{k-j-1}(a)$; thus the number of solutions of (6) which also satisfy (7) is bounded above by $\phi^{k-j-1}(a)$.

Now, suppose that $\left(n_{j}, \varphi\left(n_{j}\right)\right)=1$, let $l_{j+1}, \cdots, l_{k}$ be a set of integers that satisfies (6) and (7), and let $b_{i}=1+l_{i}$ for $i=j+1, \cdots, k$. 
Then we have

$$
\lambda\left(n_{j}\right)=\sum_{n_{k-j} \in A\left(n_{j}\right)} 1=\sum_{\left\{b_{j+1}, \cdots, b_{k}\right\}} \pi\left(\frac{x}{n_{j}} ; n_{j}, b_{j+1}, \cdots, b_{k}\right)
$$

where $\left\{b_{j+1}, \cdots, b_{k}\right\}$ runs over the sets of integers we get when $\left\{l_{j+1}, \cdots, l_{k}\right\}$ runs over the distinct solutions of (6) which satisfy (7). Lemma 1 will be applicable here if

$$
\log _{k-j+1}\left(x / n_{j}\right) \geqq n_{j},
$$

but this is the case if $n_{j} \leqq \log _{k+1} x$ since

$$
\log _{k-j+1}\left(x / n_{j}\right) \geqq \log _{k-j+1}\left(x / \log _{k+1} x\right) \geqq \log _{k}\left(x / \log _{k+1} x\right) \geqq \log _{k+1} x \geqq n_{j}
$$

for $x \geqq c_{1}, c_{1}$ being a constant that depends on $k$. Consequently if $j \leqq k-2$ and $n_{j} \leqq \log _{k+1} x$ then

$$
\lambda\left(n_{j}\right)=\frac{a\left(n_{j}\right)}{\varphi^{k-j}\left(n_{j}\right)} \frac{x}{n_{j}} \frac{\log _{2}^{k-j-1} x}{\log x}\left[1+\mathcal{O}\left(\frac{\log _{3} x}{\log _{2} x}\right)\right]
$$

where $a\left(n_{j}\right)$ is an integer such that $a\left(n_{j}\right) \leqq k \varphi^{k-j-1}\left(n_{j}\right)$. Lemma 2 implies that (8) also holds if $j=k-1$.

If we take $j=1$ we have, by (8)

$$
\begin{aligned}
& \sum_{n_{1} \leqq \log _{k+1} x} \sum_{n_{k-1} \in A\left(n_{1}\right)} 1 \\
& \quad=\sum_{n_{1} \leqq \log _{k+1} x} \frac{a\left(n_{1}\right)}{\varphi^{k-1}\left(n_{1}\right)} \frac{x}{n_{1}} \frac{\log _{2}^{k-2} x}{\log x}\left[1+\mathcal{O}\left(\frac{\log _{3} x}{\log _{2} x}\right)\right] .
\end{aligned}
$$

Set

$$
\alpha_{k}=\sum_{n_{1}} \frac{a\left(n_{1}\right)}{\phi^{k-1}\left(n_{1}\right) n_{1}} .
$$

Since $a\left(n_{1}\right) \leqq k \varphi^{k-2}\left(n_{1}\right)$ this infinite series converges. Furthermore, $\alpha_{k} \neq 0$. For, since $n_{1}$ is a prime, say $n_{1}=p_{1}, a\left(p_{1}\right)$ is the number of solutions of the congruence $l_{2} \cdots l_{k} \equiv 1 \bmod p_{1}$ such that $\left(1+l_{i}, p_{1}\right)=1$ for $i=2, \cdots, k$. Since the set of values $l_{2}=l_{3}=\cdots=l_{k}=1$ satisfies these conditions we have $a\left(n_{1}\right)=a\left(p_{1}\right)>0$ for $p_{1} \geqq 3$, i.e. $\alpha \alpha_{k} \neq 0$. In short, the left hand side of (9) is equal to

$$
\frac{\alpha_{k} x \log _{2}^{k-2} x}{\log x}\left[1+\mathcal{O}\left(\frac{1}{\log _{k+1} x}\right)\right],
$$

where $\alpha_{k}$ is a positive constant.

If $2 \leqq j \leqq k-1$ then we have, by (8)

$$
\sum_{n_{j} \leqq \log _{k+1} x} \sum_{n_{k-j} \in A\left(n_{j}\right)} 1=\mathcal{O}\left[\frac{x \log _{2}^{k-3} x}{\log x}\right] .
$$


Formula (4) follows from this and preceding result.

The main problem that remains is that of finding a reasonable bound for the quantity $S$ where

and $z=\left(\log _{k+1} x\right)^{1 / k}$.

$$
S=\sum_{p_{1}>z} \sum_{n_{k-1} \in A\left(p_{1}\right)} 1
$$

To that end, fix $p_{1}$ and define $B\left(p_{1}, i\right)$ to be the set of integers $p_{2} \cdots p_{k}$ in $A\left(p_{1}\right)$ such that $i-1$ of the prime factors of $p_{2} \cdots p_{k}$ are less than $p_{1}$ and $k-i$ are greater than $p_{1}$, for $i=1, \cdots, k$. Then

$$
S=\sum_{p_{1}>z} \sum_{i=1}^{k} \sum_{n_{k-1} \in B\left(p_{1}, i\right)} 1
$$

If we fix $p_{1}$ and $i$, where $2 \leqq i \leqq k-1$, we have

$$
\sum_{n_{k-1} \in B\left(p_{1}, i\right)} 1=\sum_{p_{2} \cdots p_{i}} \sum_{p_{i+1} \cdots p_{k} \in O} 1
$$

where each of the prime factors of $p_{2} \cdots p_{i}$ is less than $p_{1}$ and $C$, which depends on $p_{1}, \cdots$, and $p_{i}$, is the set of integers $p_{i+1} \cdots p_{k}$ such that $p_{j}>p_{1}$ for $j>i, p_{1} \cdots p_{i} p_{i+1} \cdots p_{k}$ is square free and less than or equal to $x$, and $\varphi\left(p_{1} \cdots p_{k}\right)+1 \equiv 0 \bmod p_{1}$. Similar statements can be made when $i=1$. If we fix $p_{1}, \cdots$, and $p_{i}$ our problem then is that of finding primes $p_{i+1}, \cdots$, and $p_{k}$ such that

$$
\left(p_{i+1}-1\right) \cdots\left(p_{k}-1\right) \equiv l \bmod p_{1}
$$

where $l$ is an integer, relatively prime to $p_{1}$, that depends on $p_{1}, \cdots$, and $p_{i}$. Let $D$ be the set of $(k-i)$-tuples

$$
\left\{\left(b_{i+1}, \cdots, b_{k}\right):\left(b_{i+1}-1\right) \cdots\left(b_{k}-1\right) \equiv l \bmod p_{1}, 0 \leqq b_{j} \leqq p_{1}\right\} .
$$

Then, holding $p_{1}, p_{2}, \cdots$, and $p_{i}$ fixed we have

$$
\sum_{p_{i+1} \cdots p_{k} \in O} 1 \leqq \sum_{\left(b_{i+1}, \cdots, b_{k}\right) \in D} \sum_{\substack{p_{1} \cdots p_{k} \leq x \\ p_{1}<p_{j}=b_{j} \bmod p_{1}, j>i}} 1
$$

If we fix $\left(b_{i+1}, \cdots, b_{k}\right)$ and let $\left(b_{i+1}^{\prime}, \cdots, b_{k}^{\prime}\right)$ run over the $(k-i)$-tuples we get by permuting the integers $b_{i+1}, \cdots$, and $b_{k}$ we have

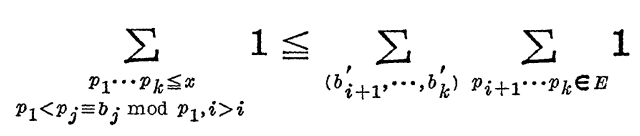

where $E$ is the set of integers

$$
\begin{aligned}
\left\{p_{i+1} \cdots p_{k}: p_{1} \cdots p_{i}\right. & \cdots p_{k} \leqq x, p_{1}<p_{i+1}<\cdots \\
& \left.<p_{k}, p_{j} \equiv b_{j}^{\prime} \bmod p_{1}, j>i\right\}
\end{aligned}
$$

Now, fix $\left(b_{i+1}^{\prime}, \cdots, b_{k}^{\prime}\right)$. If $p_{i+1} \cdots p_{k}$ is in $E$ it follows, by induction, that 


$$
p_{k-r} \leqq\left(x / p_{1} \cdots p_{k-r-1}\right)^{\frac{1}{r+1}}=t(r)
$$

since $p_{1} \cdots p_{k-1} p_{k} \leqq x$ and $p_{k-(r+1)} \leqq p_{k-r}$ for $r=0,1, \cdots, k-(i+1)$. Consequently we have

$$
\sum_{p_{i+1} \cdots p_{k} \in E} 1 \leqq \sum_{p_{i+1}}^{\prime} \cdots \sum_{p_{k-r}}^{\prime} \cdots \sum_{p_{k-1}}^{\prime} \pi\left(\frac{x}{p_{1} \cdots p_{k-1}} ; p_{1}, b_{k}^{\prime}\right)
$$

where the prime on the summation symbol indicates that $p_{1}<p_{k-r} \leqq t(r)$ and $p_{k-r} \equiv b_{k-r}^{\prime} \bmod p_{1}$ for $r=1, \cdots, k-(i+1)$.

Split the quantity on the right hand side of (11) into two sums, $\sum_{1}$ and $\Sigma_{2}$. The index of summation of $\Sigma_{1}$ will be those integers $p_{1} \cdots p_{k-1}$ such that $p_{1}^{2} p_{2} \cdots p_{k-1} \leqq x^{1-\delta}$, where $\delta$ is a positive number that will be chosen later; the index of summation of $\Sigma_{2}$ will be those $p_{1} \cdots p_{k-1}$ such that $p_{1}^{2} p_{2} \cdots p_{k-1}>x^{1-\delta}$.

We have, by Lemmas 3 and 4 ,

$$
\begin{aligned}
\sum_{1} & \leqq \sum_{p_{i+1}}^{\prime} \cdots \sum_{p_{k-1}}^{\prime} \frac{c_{2} x}{\varphi\left(p_{1}\right) p_{1} \cdots p_{k-1}} \frac{1}{\log \left(x / p_{1}^{2} p_{2} \cdots p_{k-1}\right)} \\
& \leqq \frac{c_{3}}{\varphi\left(p_{1}\right) p_{1} \cdots p_{i}} \frac{x}{\log x} \prod_{j=i+1}^{k-1}\left(\sum_{\substack{p_{j} \leqq x \\
p_{j} \equiv b_{i}^{\prime} \text { mod } p_{1}}} \frac{1}{p_{j}}\right) \\
& \leqq \frac{c_{4}}{\varphi^{k-i}\left(p_{1}\right) p_{1} \cdots p_{i}} \frac{x}{\log x} \log _{2}^{k-i-1} x,
\end{aligned}
$$

where $c_{3}, c_{4}, \cdots$ are constants that depend on $k$ and $\delta$. If we sum on $\left(b_{i+1}^{\prime}, \cdots, b_{k}^{\prime}\right)$ we have

$$
\Sigma_{3}=\sum_{\left(b_{i+1}^{\prime}, \cdots, b_{k}^{\prime}\right)} \sum_{1} \leqq \frac{c_{5}}{\varphi^{k-i}\left(p_{1}\right) p_{1} \cdots p_{i}} \frac{x \log _{2}^{k-i-1} x}{\log x}
$$

since there are at most $(k-i)$ ! permutations of $b_{i+1}, \cdots, b_{k}$. Summing on $\left(b_{i+1}, \cdots, b_{k}\right)$ yields

$$
\sum_{4}=\sum_{\left(b_{j+1}, \cdots, b_{k}\right) \in D} \sum_{3} \leqq \frac{c_{6}}{\varphi\left(p_{1}\right) p_{1} \cdots p_{i}} x \frac{\log _{2}^{k-i-1} x}{\log x},
$$

for there are at most $\varphi^{k-i-1}\left(p_{1}\right)(k-i)$-tuples in the set $D$. Since $p_{j}<p_{1}$ for $j=2, \cdots, i$ we have

$$
\begin{aligned}
\sum_{5} & =\sum_{p_{2} \cdots p_{i}} \sum_{4} \leqq \frac{c_{6}}{\varphi\left(p_{1}\right) p_{1}}\left(\sum_{p<p_{1}} \frac{1}{p}\right)^{i-1} x \frac{\log _{2}^{k-i-1} x}{\log x} \\
& \leqq \frac{c_{7} \log _{2}^{i-1} p_{1}}{\varphi\left(p_{1}\right) p_{1}} \frac{x \log _{2}^{k-i-1} x}{\log x}
\end{aligned}
$$

Since we have the restriction $i \leqq k-1$, we have

$$
\Sigma_{6}=\sum_{i=1}^{k-1} \sum_{15} \leqq c_{8} \frac{\log _{2}^{k-2} p_{1}}{p_{1} \varphi\left(p_{1}\right)} \frac{x \log _{2}^{k-2} x}{\log x}
$$


Finally, since $p_{1}>z$, it follows that

$$
\sum_{p_{1}>z} \sum_{6} \leqq c_{9} \frac{x}{\log x} \frac{\log _{2}^{k-2} x}{\sqrt{z}}
$$

In short, we have

$$
\sum_{p_{1}>x} \sum_{i=1}^{k-1} \sum_{n_{k-1} \in B\left(p_{1}, i\right)} 1 \leqq c_{9} \frac{x}{\log x} \frac{\log _{2}^{k-2} x}{\sqrt{z}}
$$

where the summation is restricted to those integers $p_{1}$ and $n_{k-1}=$ $p_{2} \cdots p_{k}$ for which $p_{1}^{2} p_{2} \cdots p_{k-1} \leqq x^{1-\delta}$.

Let us return to (11) and deal with $\sum_{2}$, i.e. with those integers $p_{1} \cdots p_{k}$ where $p_{1}^{2} p_{2} \cdots p_{k-1}>x^{1-\delta}$. Under these circumstances we have

$$
\pi\left(\frac{x}{p_{1} \cdots p_{k-1}} ; p_{1}, b_{k}^{\prime}\right) \leqq \pi\left(p_{1} x^{\delta} ; p_{1}, b_{k}^{\prime}\right) \leqq \frac{c_{2} x^{\delta}}{\log x^{\delta}} \leqq c_{3} x^{\delta} .
$$

Since we also have, for $0 \leqq \alpha<1$,

$$
\sum_{\substack{p_{1}<p \leqq y \\ p \equiv b \bmod p_{1}}} \frac{1}{p^{\alpha}} \leqq c_{4} \frac{y^{1-\alpha}}{p_{1}}
$$

we can prove, by induction, that

$$
\sum_{2} \leqq c_{5} \sum_{p_{i+1}}^{\prime} \cdots \sum_{p_{k-r}}^{\prime} \frac{x^{\delta+1-\frac{1}{r}}}{p_{1}^{r-1}\left(p_{1} \cdots p_{k-r}\right)^{1-(1 / r)}} .
$$

Thus if follows that

$$
\sum_{2} \leqq \frac{c_{6}}{p_{1}^{k-i-1}} \frac{x^{\delta+\beta}}{\left(p_{1} \cdots p_{i}\right)^{\beta}}
$$

where $\beta=\beta(i)=1-(k-i)^{-1}$. If we now sum on $\left(b_{i+1}^{\prime}, \cdots, b_{k}^{\prime}\right)$ and $\left(b_{i+1}, b_{k}\right)$ we obtain a quantity that is bounded above by

$$
c_{7} x^{\delta+\beta} /\left(p_{1} \cdots p_{i}\right)^{\beta} \text {. }
$$

Omit the summation on $i$ for the moment, and divide the sum

$$
\sum_{p_{1}} \sum_{p_{2} \cdots p_{i}} x^{\delta+\beta} /\left(p_{1} \cdots p_{i}\right)^{\beta}
$$

into two parts, the first, $\sum_{7}$, being that part where $p_{1} \leqq x^{\varepsilon}$, the second, $\sum_{8}$, being that part where $p_{1}>x^{\varepsilon}, \varepsilon$ being a positive number that will be chosen later. We have, since $p_{j}<p_{1}$ for $j=2, \cdots, i$,

$$
\sum_{7}=\sum_{x<p_{1} \leqq x^{e}} \sum_{p_{2} \cdots p_{i}} \frac{x^{\delta+\beta}}{\left(p_{1} \cdots p_{i}\right)^{\beta}} \leqq\left(\sum_{p_{1} \leqq x^{\varepsilon}} \frac{1}{p_{1}^{\beta}}\right)^{i} x^{\delta+\beta} \leqq c_{8} x^{\lambda}
$$


where $\lambda=i(1-\beta) \varepsilon+\delta+\beta$. Furthermore since

$$
x \geqq p_{1} \cdots p_{i} p_{i+1} \cdots p_{k} \geqq p_{1}^{k-i+1} p_{2} \cdots p_{i}
$$

it follows that

$$
\begin{aligned}
\sum_{8} & \leqq \sum_{p_{1}>x^{\varepsilon}} \frac{1}{p_{1}^{\beta}} \sum_{p_{2} \cdots p_{i} \leqq x / p_{1}^{k-i+1}} \frac{x^{\delta+\beta}}{\left(p_{2} \cdots p_{i}\right)^{\beta}} \\
& \leqq c_{9}\left(\sum_{p_{1}>x^{\varepsilon}} \frac{1}{p_{1}^{2}}\right) x^{\delta+\beta+1-\beta} \leqq c_{10} x^{1+\delta-\varepsilon} .
\end{aligned}
$$

Set $\varepsilon=1 /(2 k)$ and $\delta=1 /(4 k)$. Then $1+\delta-\varepsilon=1-(4 k)^{-1}, \lambda \leqq 1-$ $(4 k)^{-1}$, and

$$
\Sigma_{7}+\Sigma_{8} \leqq c_{11} x^{1-\frac{1}{4 k}}
$$

A summation on the $i$ 's yields the inequality

$$
\sum_{p_{1}>z} \sum_{i=1}^{k-1} \sum_{n_{k-1} \in B\left(p_{1}, i\right)} 1 \leqq c_{12} x^{1-\frac{1}{4 k}},
$$

where the summation is restricted to those integers $p_{1}$ and $n_{k-1}=p_{2} \cdots p_{j}$ for which $p_{1}^{2} p_{2} \cdots p_{k-1}>x^{1-\delta}$.

If we return to (10) we see that we must find a bound for that part of $S$ corresponding to $i=k$. If we have $n_{k-1}=p_{2} \cdots p_{k}$ in $B\left(p_{1}, k\right)$ then, by definition, $p_{j}<p_{1}$ for $j=2, \cdots, k$ and

$$
\left(p_{2}-1\right) \cdots\left(p_{k}-1\right)-1 \equiv 0 \bmod p_{1} \text {. }
$$

Once again we have a two way split. On one hand we have

$$
\sum_{z<p_{1} \leqq x^{1 / k}} \sum_{n_{k-1} \in B\left(p_{1}, k\right)} 1 \leqq \sum_{p_{1} \leqq x^{1 / k}} \pi\left(p_{1}\right)^{k-2} \leqq c_{1} x^{1-1 / k},
$$

for the obvious reasons. On the other hand, if $p_{1}>x^{1 / k}$ then $p_{2} \cdots p_{k} \leqq x^{1-1 / k}$ and

$$
\sum_{p_{1}>x^{1 / k}} \sum_{n_{k-1} \in B\left(p_{1}, k\right)} 1 \leqq \sum_{p_{2} \cdots p_{k} \leqq x^{1-1 / k}} \sum_{\substack{p_{1}>x^{1 / k} \\ p_{1} \mid\left(\varphi\left(p_{2} \cdots p_{k}\right)-1\right)}} 1
$$

Since the number of prime divisors of $\varphi\left(p_{2} \cdots p_{k}\right)-1$ which are greater than $x^{1 / k}$ does not exceed $k$ this last double sum is bounded above by $k x^{1-1 / k}$. Therefore, we can conclude that

$$
\sum_{p_{1}>z} \sum_{n_{k-1} \in B\left(p_{1}, k\right)} 1 \leqq c_{2} x^{1-1 / k}
$$

Let us assemble our results. Items (10), (12), (13), and (14) imply that

$$
S=\mathscr{O}\left[\frac{x}{\log x} \frac{\log _{2}^{k-2} x}{\sqrt{z}}\right]
$$


Relations (3), (4) and (15) yield

$$
\Phi(k, x)=\alpha_{k} x \frac{\log _{2}^{k-2} x}{\log x}\left[1+\mathcal{O}\left(\frac{1}{\sqrt{z}}\right)\right]
$$

where $z=\left(\log _{k+1} x\right)^{1 / k}$. Since $\Phi^{\prime}(k, x)$ was defined to be the number of positive square free integers $m$ less than or equal to $x$ which have $k$ prime factors and which have a factor in common with $\varphi(m)+1$, and since $\Phi(k, x)$ was defined to be the number of odd integers counted

by $\Phi^{\prime}(k, x)$ we have, for $k>2$,

$$
\Phi^{\prime}(k, x)=\Phi(k, x)+\Phi(k-1, x / 2) \sim\left(\alpha_{k} x \log _{2}^{k-2} x\right) / \log x .
$$

If $k=2$ then

$$
\Phi^{\prime}(2, x)=\Phi(2, x)+\pi(x / 2) \sim\left(\alpha_{2}+1 / 2\right) x / \log x .
$$

These are the results we set out to prove.

\section{BIBLIOGRAPHY}

1. K. Prachar, Primzahlverteihung, Springer, 1957.

2. E. M. Wright, A simple proof of a theorem of Landau, Proc. Edinburgh Math. Soc. (2) 9 (1954), 87-90.

Received August 25, 1965. This research was supported, in part, by funds received under NSF grant GP-3933.

University of California, Los ANgeles 



\section{PACIFIC JOURNAL OF MATHEMATICS}

\section{EDITORS}

\author{
H. SAMELSON \\ Stanford University \\ Stanford, California \\ J. P. JANS \\ University of Washington \\ Seattle, Washington 98105
}

\author{
J. DUGUNDJI \\ University of Southern California \\ Los Angeles, California 90007 \\ RICHARD ARENS \\ University of California \\ Los Angeles, California 90024
}

\section{ASSOCIATE EDITORS}
E. F. BECKENBACH
B. H. NeumanN
F. WOLF
K. YOSIDA

\section{SUPPORTING INSTITUTIONS}

\author{
UNIVERSITY OF BRITISH COLUMBIA \\ CALIFORNIA INSTITUTE OF TECHNOLOGY \\ UNIVERSITY OF CALIFORNIA \\ MONTANA STATE UNIVERSITY \\ UNIVERSITY OF NEVADA \\ NEW MEXICO STATE UNIVERSITY \\ OREGON STATE UNIVERSITY \\ UNIVERSITY OF OREGON \\ OSAKA UNIVERSITY \\ UNIVERSITY OF SOUTHERN CALIFORNIA
}

STANFORD UNIVERSITY

UNIVERSITY OF TOKYO

UNIVERSITY OF UTAH

WASHINGTON STATE UNIVERSITY

UNIVERSITY OF WASHINGTON

AMERICAN MATHEMATICAL SOCIETY CHEVRON RESEARCH CORPORATION TRW SYSTEMS

NAVAL ORDNANCE TEST STATION 


\section{Pacific Journal of Mathematics}

\section{Vol. 19, No. 1 \\ May, 1966}

A. R. Brodsky, The existence of wave operators for nonlinear equations... 1

Gulbank D. Chakerian, Sets of constant width................... 13

Robert Ray Colby, On indecomposable modules over rings with minimum condition....................................... 23

James Robert Dorroh, Contraction semi-groups in a function space ....... 35

Victor A. Dulock and Harold V. McIntosh, On the degeneracy of the Kepler

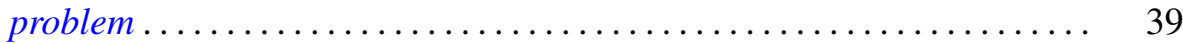

James Arthur Dyer, The inversion of a class of linear operators ......... 57

N. S. Gopalakrishnan and Ramaiyengar Sridharan, Homological dimension of Ore-extensions ................................. 67

Daniel E. Gorenstein, On a theorem of Philip Hall ................. 77

Stanley P. Gudder, Uniqueness and existence properties of bounded observables..................................... 81

Ronald Joseph Miech, An asymptotic property of the Euler function ....... 95

Peter Alexander Rejto, On the essential spectrum of the hydrogen energy and related operators ............................... 109

Duane Sather, Maximum and monotonicity properties of initial boundary

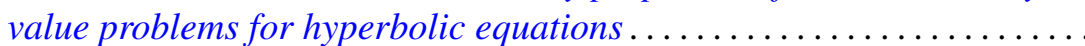

Peggy Strait, Sample function regularity for Gaussian processes with the parameter in a Hilbert space ........................... 159

Donald Reginald Traylor, Metrizability in normal Moore spaces ........... 175

Uppuluri V. Ramamohana Rao, On a stronger version of Wallis' formula ...............................

Adil Mohamed Yaqub, Some classes of ring-logics....... 